\title{
One-stage correction for blepharophimosis syndrome
}

\begin{abstract}
Purpose To classify the severity of blepharophimosis, describe associated features and their effects on the incidence of amblyopia and to recommend guidelines for surgical treatment and management of surgical complications.

Methods The case records of 23 patients with blepharophimosis syndrome were examined retrospectively. Patients' photographs and measurements were reviewed to analyse the severity of blepharophimosis, surgical techniques undertaken, surgical outcomes, and complications. Statistical analyses were performed using paired-sample $t$-tests to evaluate the surgical outcome and Spearman correlation to examine the influence of blepharophimosis on the interpalpebral fissure height (PFH).

Results Eighteen out of 23 (78\%) patients underwent one-stage surgery before the age of 5 years. About $31 \%$ of these patients had amblyopia. Only two patients had a blepharophimosis ratio greater than 1.5 as poor result. Two out of $18(\mathbf{1 1 \%})$ patients with PFHs more than $2 \mathrm{~mm}$ needed a repeat operation, but all five (100\%) patients with $\mathrm{s}$ less than $2 \mathrm{~mm}$ (very severe ptosis) needed repeat operations.

Conclusions The one-stage corrective procedure provided acceptable results both in function and cosmesis. However, patients with very severe ptosis required multiple stages of reconstruction for ptosis correction at an earlier age, after which correction of telecanthus and small horizontal palpebral fissure length followed at an older age.
\end{abstract}

Eye (2008) 22, 380-388; doi:10.1038/sj.eye.6702644; published online 17 November 2006

Accepted in revised form:

1 October 2006

Published online:

17 November 2006
S-Y Wu, L Ma, Y-J Tsai and JZ-C Kuo

\section{Introduction}

Blepharophimosis-ptosis-epicanthus inversus (BPES) syndrome is a rare congenital disorder and occurs either as an autosomal dominant trait or sporadically. ${ }^{1-6}$ In 1971, Kohn and Romano $^{2}$ described the tetrad of this syndrome, which includes blepharophimosis, blepharoptosis, epicanthus inversus, and telecanthus. It is also associated with ocular, lacrimal, nasal, and auricular anomalies. ${ }^{4-6}$ There are two subtypes of this syndrome proposed by Zlotogora et al: ${ }^{3}$ type I is more prevalent and affected female subjects are infertile and type II is transmitted both in affected male and female subjects. ${ }^{3}$

Blepharophimosis syndrome (BPES) has a great impact on a patient's functional status and may cause poor visual development. ${ }^{7}$ Although previous studies describe the demography and aetiology of children with blepharophimosis syndrome, ${ }^{1-6,8-11}$ there is a little information in the ophthalmic literature regarding the severity of the syndrome, surgical outcomes, and complications. ${ }^{12-17}$ Our study focuses on a blepharophimosis syndrome cohort undergoing one-stage surgical correction in a tertiary referral oculoplastic centre.

\section{Methods}

This is a retrospective, interventional case series report. Medical records of all patients who underwent one-stage surgical correction for blepharophimosis syndrome at Chang-Gung Memorial Hospital between 1 January 1984 and 31 December 2004 were reviewed. The study complied with the policies of the institutional review board, and permission was granted to conduct the study. Data regarding visual acuity, refraction, results of orthoptic examinations, vertical interpalpebral fissure height (IPFH), horizontal palpebral fissure length (HPFL), inner intercanthal distance (IICD) were 
recorded and analysed. Amblyopia was defined as the best refracted visual acuity of 20/40 or less in the affected eye and two lines of difference on the Snellen chart. ${ }^{7,18}$ The severity of ptosis was classified as mild, moderate or severe, based on the criteria described by Freuh. ${ }^{19}$ We also defined very severe ptosis as less than $2 \mathrm{~mm}$ of palpebral aperture. Levator function was measured as the maximum lid excursion from maximal down gaze to up gaze, with frontal function abolished. This was recorded as poor if it was less than $4 \mathrm{~mm}$, as fair if it was between 4 and $8 \mathrm{~mm}$ and good if it was more than $8 \mathrm{~mm}$.

According to the normal range for IICD and interorbital distance in the Chinese population, ${ }^{20-22}$ the ratio between the IICD and HPFL was approximately $1-1.2$. We defined a ratio from 1.3 to 1.5 as mild blepharophimosis, $1.5-1.8$ as moderate blepharophimosis and greater than 1.8 as severe blepharophimosis. All patients had the same senior surgeon (Dr Lih Ma) and surgery was performed with the patient under general anaesthesia. The criteria for surgical results for blepharophimosis was classified as good if the ratio between the IICD and HPFL was less than 1.3, as suboptimal if the ratio was between 1.3 and 1.5 , as poor if the ratio was more than 1.5. The surgical outcome for ptosis was judged as good, moderate, or poor, based on the criteria described by Manners. ${ }^{23}$ A poor outcome often led to the decision to reoperate.

\section{Surgical technique}

One-stage surgical correction consisted of lateral canthotomy, medial canthoplasty, transnasal wiring for medial canthal tendon shortening, and frontalis suspension using donor fascia lata or levator resection.
The skin was marked with the modified 'Mustarde technique' figure for correcting epicanthus inversus, and with lid crease lines for levator resection or frontalis suspension. ${ }^{16,17}$ For Mustarde technique, the desired final position of the canthus in most patients was approximately half the distance from the centre of the pupil to the centre of the nasal bridge. As the IICD of Asian children is nearly $28 \mathrm{~mm},{ }^{21,22}$ we used $24 \mathrm{~mm}$ as the desired intercanthal distance for all patients during the operation (Figure 1a), allowing for overcorrection.

Our first step was to perform a lateral canthotomy of $5 \mathrm{~mm}$ to elongate the narrow horizontal palpebral fissure. Then, we sutured the conjunctiva to the eyelid raw edge with polyglactin 6-0 (Vicryl) to prevent adhesion (Figure 1b). The modified Mustarde flaps were incised and the fibrous tissue beneath the fold was removed. The entire medial canthal area was mobilised by making a vertical incision into the periosteum just medial to the anterior lacrimal crest. The medial canthal tendon was clearly identified. An anchorage hole for the wire was located superior to the level of medial canthal tendon and carefully prepared with an otology burr (Figure 1c). The wire passer required a 19-G needle. The needle on the wire suture imbricated the temporal aspect of the medial canthal tendon. The 2-0 surgical wire was passed to the contralateral side by a curved-angle needle. The contralateral medial canthal tendon was imbricated with the wire and tightening of the suture pulled both tendons toward the midline. The wires were then twisted over the intervening bone. The cut ends were placed inside the contralateral hole so there was less wire under the palpebral skin surface. The surgical wire was secured to two sides of medial canthal tendon so we could adjust the distance between two sides (Figure 1d). The skin of
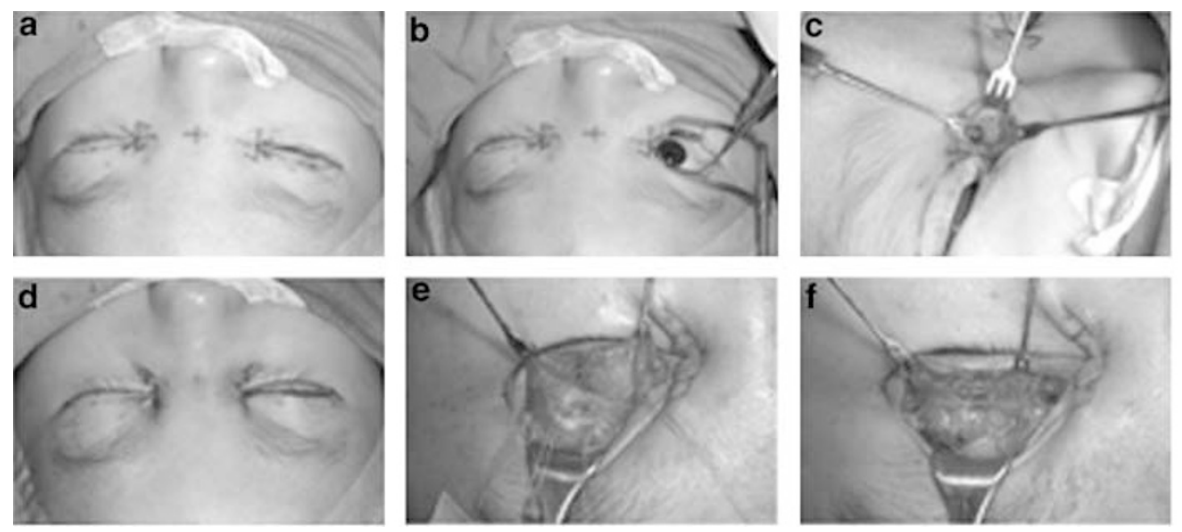

Figure 1 Operative procedure. (a) Skin markings. (b) Lateral canthotomy and suturing the conjunctiva to the eyelid cutting edge with polyglactin 6-0 (Vicryl) to prevent adhesion. (c) Skin hooks gently retract the flaps to expose the medial canthal structures. Fibrous tissues are exposed and excised. An anchorage hole for the wire is located superior to the level of medial canthal tendon. (d) The surgical wire is secured to two sides of the medial canthal tendon so as to adjust the distance between two sides. (e) Dissection of the levator aponeurosis from the septum and conjunctiva to show Whitnall's ligament. Placement of a double-armed, 4-0 Vicryl suture at the tarsus through the aponeurosis above Whitnall's ligament. (f) Removing the redundant levator aponeurosis. 
medial commissure was sutured to the deep tissue and desired canthal skin point with 4-0 Vicryl. The skin flaps were trimmed and sewn into position with a 6-0 Vicryl suture. Our methods emphasised not only the design of incision, but also firm and symmetrical attachments to shorten the lengthened medial canthal tendon to its insertion. All of these procedures were performed depending on the degree of severity of blepharophimosis syndrome.

The surgical approach to congenital ptosis is based on the amount of levator function. In all cases, the distance between the upper lid margin and the papillary reflex was $1 \mathrm{~mm}$ or less, with poor levator function of $4 \mathrm{~mm}$ or less, requiring frontalis suspension as the operation of choice. A double rhomboid technique with freeze-dried human cadaveric fascia was used in almost all patients. ${ }^{24}$ Only five patients underwent external maximal levator resection. The surgical technique used was essentially the same as that described by Mauriello et al ${ }^{25}$ in 1985, with modification. ${ }^{25}$ We did not cut the medial and lateral horns; we separated the levator aponeurosis and Muller's muscle from conjunctiva using scissors. The under surface of the levator aponeurosis was then dissected superiorly, nearly to the fornix. The septum above levator aponeurosis was opened and dissected superiorly to just above Whitnall's ligament (Figure 1e). A double-armed, 4-0 polyglactin suture was advanced to the levator approximately $2 \mathrm{~mm}$ below the superior tarsal border. Three or four sutures were placed to reform the eyelid fissure shape to a more normal shape (Figure 1f). The chromic suture was tied and 7-0 Vicryl skin-toaponeurosis-to-skin sutures were placed to create a skin fold.

\section{Statistical analyses}

Statistical analyses were performed using paired-sample $t$-tests to evaluate preoperative and postoperative IICD and HPFL data. Spearman correlation was used to examine the influence of preoperative IICD, HPFL, and blepharophimosis ratio on the IPFH.

\section{Results}

Twenty-four patients were diagnosed with blepharophimosis syndrome and underwent one-stage correction for blepharophimosis syndrome. One patient died of malignant hyperthermia and was excluded from this study. Fourteen $(60.9 \%)$ patients were female and nine $(39.1 \%)$ were male. Nine patients $(39.1 \%)$ had a positive family history of blepharophimosis syndrome (BPES), with a 7-2 predominance on the father's side. Eighteen $(78 \%)$ patients presented before the age of 5 years. Refractive data was obtained for 17 patients and five (29\%) patients had amblyopia. Pertinent data are shown in Table 1.

Patient age at surgery ranged from 16 months to 20 years (mean, 5.1 years). Eighteen of 23 (78\%) patients underwent one-stage surgery before the age of 5 years. The minimal postoperative observation period was 6 months, and the maximum postoperative follow-up was 228 months (mean, 50.43 months). Seventeen patients (74\%) with severe blepharophimosis underwent lateral canthotomy, transnasal wiring, medial canthoplasty, and ptosis surgeries in one stage (Table 2). The surgical time for frontalis suspension group was 165-315 min (mean, $230 \mathrm{~min}$ ) and only medial canthoplasty and frontalis sling was $165 \mathrm{~min}$. The mean surgical time for the levator resection group was $258 \mathrm{~min}$ (range, $250-270 \mathrm{~min}$ ). The mean IICD improved from $38.17 \mathrm{~mm}$ preoperatively to $31.52 \mathrm{~mm}$ postoperatively, with a mean difference of $6.65 \mathrm{~mm}(P<0.0001)$. The normal HPFL for adults is 25-30 mm. The mean HPFL improved from $18.96 \mathrm{~mm}$ preoperatively (range, $13-25 \mathrm{~mm}$ ) to $25.93 \mathrm{~mm}$ postoperatively (range, $20-35 \mathrm{~mm}$ ), with a mean difference of $7.03 \mathrm{~mm}(P<0.0001)$. Sixteen of $23(70 \%)$ patients had good results, with a blepharophimosis ratio of less than 1.3. Only two of $23(9 \%)$ patients had ratios of greater than 1.5 , a poor outcome (Table 3 ). The ratio did not improve for the patient who underwent only medial canthoplasty (patient 8) (Figure 2).

Twenty (87\%) patients had severe bilateral ptosis with palpebral apertures of less than $4 \mathrm{~mm}$. No patient had asymmetric ptosis ( $2 \mathrm{~mm}$ or more). Eighteen patients underwent frontalis suspensions with donor fascia lata and five patients underwent external maximal levator resection. In the frontalis suspension group, only one of 18 patients had a good outcome (patient 8) (Figure 2). Ten patients had moderate outcomes. Seven (39\%) patients had poor outcomes. The main reason for suboptimal or poor outcomes was persistently low upper eyelid. Of the seven patients with poor outcomes, five $(71 \%)$ had very severe ptosis and two (29\%) had severe ptosis. Of the two patients with severe ptosis, one required reoperation for asymmetrical lid position and the other, for residual ptosis. One of the five patients with a poor outcome (patient 6) underwent frontalis suspension with Supramid (S Jackson Inc., Alexandria, VA, USA) at the age of 8 months, before the one-stage correction (Figure 3). In the levator resection group, all patients had moderate outcomes (Figure 4) and no patient required re-operation. Data are summarised in Table 4.

Preoperatively smaller horizontal palpebral lengths had smaller palpebral apertures (right eye, $r=0.4997$, $P=0.0152$; left eye, $r=0.4839, P=0.0193)$. No correction was found for internal intercanthal distances or IPFHs before surgery. Smaller blepharophimosis ratios tended to have higher palpebral apertures preoperatively (right 
Table 1 General information for 23 patients with blepharophimosis syndrome

\begin{tabular}{|c|c|c|c|c|c|c|c|c|}
\hline \multirow[t]{2}{*}{ No/sex } & \multirow{2}{*}{$\begin{array}{l}\text { Family } \\
\text { history }\end{array}$} & \multirow{2}{*}{$\begin{array}{c}\text { Age at } \\
\text { first visit }\end{array}$} & \multirow{2}{*}{$\begin{array}{c}\text { Age at } \\
\text { OP }\end{array}$} & \multirow{2}{*}{$\begin{array}{c}\text { Age at last } \\
\text { visit }\end{array}$} & \multicolumn{2}{|c|}{ Refraction (last time) } & \multirow[t]{2}{*}{ Strabismus } & \multirow[t]{2}{*}{ Amblyopia } \\
\hline & & & & & $O D$ & OS & & \\
\hline \multicolumn{9}{|l|}{$L R$ group } \\
\hline $1 / \mathrm{F}$ & $\mathrm{N}$ & $1 \mathrm{Y} 2 \mathrm{M}$ & $3 Y$ & $3 Y 6 \mathrm{M}$ & No data & No data & & \\
\hline $2 / \mathrm{F}$ & $\mathrm{N}$ & $2 Y$ & $2 Y 7 M$ & $4 \mathrm{Y} 1 \mathrm{M}$ & $(0.3 x+1.0 /-4.0 \times 165)$ & $(0.2 x+2.0 /-4.0 \times 5)$ & & $\mathrm{P}$ \\
\hline $3 / \mathrm{F}$ & $\mathrm{N}$ & $8 \mathrm{M}$ & $2 Y 5 \mathrm{M}$ & $4 \mathrm{Y} 5 \mathrm{M}$ & $(0.8 \times 0 /-5.0 \times 170)$ & $(0.4 x+1.0 /-5.5 \times 175)$ & & $\mathrm{P}$ \\
\hline $4 / \mathrm{M}$ & $\mathrm{N}$ & 1Y7M & $2 Y 5 \mathrm{M}$ & $4 Y 5 \mathrm{M}$ & $(1.0 x+1.0 /-2.25 \times 160)$ & $(0.8 x+1.0 /-3.5 \times 10)$ & & \\
\hline $5 / \mathrm{M}$ & $\mathrm{N}$ & $2 \mathrm{YMM}$ & $3 Y 7 \mathrm{M}$ & $5 \mathrm{Y} 3 \mathrm{M}$ & $(-0.25 /-2.75 \times 5)^{\mathrm{a}}$ & $(+1.5 /-2.0 \times 5)^{\mathrm{a}}$ & & \\
\hline \multicolumn{9}{|l|}{ FS group } \\
\hline $6 / \mathrm{M}$ & $\mathrm{N}$ & $2 \mathrm{M}$ & $1 Y 8 M$ & $4 Y 9 M$ & $(0 /-2.50 \times 25)^{\mathrm{a}}$ & $(-1.50 x-2.0 \times 160)^{a}$ & & \\
\hline $7 / \mathrm{F}$ & $\mathrm{N}$ & $8 \mathrm{Y} 6 \mathrm{M}$ & $8 Y 6 \mathrm{M}$ & $9 Y 6 \mathrm{M}$ & $(1.0 x-4.25 /-1.0 \times 175)$ & $(1.0 x-3.5 /-1.0 \times 0)$ & $\mathrm{X}-\mathrm{XT}$ & \\
\hline $8 / \mathrm{F}$ & $\mathrm{N}$ & $4 Y 6 \mathrm{M}$ & $4 Y 8 \mathrm{M}$ & $5 Y 4 \mathrm{M}$ & $(1.0 x-0.25 /-1.0 \times 170)$ & $(1.0 x+0.25 /-1.0 \times 10)$ & & \\
\hline $9 / \mathrm{M}$ & Father & $5 \mathrm{M}$ & $1 Y 4 \mathrm{M}$ & $10 Y$ & $(0.7 \times 0 /-1.0 \times 180)$ & $(0.7 \times 0 /-0.75 \times 0)$ & XTKr50(OP) & \\
\hline $10 / \mathrm{F}$ & $\mathrm{N}$ & $3 Y 1 M$ & $3 \mathrm{Y} 2 \mathrm{M}$ & $11 Y 3 M$ & $(1.0 x+0.50 /-2.25 \times 10)$ & $(1.0 x+0.50 /-0.5 \times 70)$ & & \\
\hline $11 / \mathrm{F}$ & Father & $20 Y$ & $20 Y 7 M$ & $21 Y 1 M$ & $(0.6 x-7.50 /-0.50 \times 175)$ & $(0.6 x-4.0 /-0.5 \times 15)$ & & \\
\hline $12 / F$ & $\mathrm{~N}$ & $4 \mathrm{Y} 1 \mathrm{M}$ & $4 \mathrm{Y} 1 \mathrm{M}$ & $4 \mathrm{Y} 11 \mathrm{M}$ & $(0.2 x+2.25 /-4.0 \times 175)$ & $(0.5 x+2.25 /-2.5 \times 180)$ & & $\mathrm{P}$ \\
\hline $13 / \mathrm{F}$ & $\mathrm{N}$ & $2 \mathrm{Y} 2 \mathrm{M}$ & $2 Y 3 \mathrm{M}$ & $3 Y 7 \mathrm{M}$ & No data & No data & & \\
\hline $14 / \mathrm{M}$ & Father & $1 Y 3 M$ & $2 \mathrm{YM}$ & $5 Y 4 \mathrm{M}$ & $(1.0 \times 0.75 /-3.0 \times 0)$ & $(1.0 x+0.5 /-3.0 \times 0)$ & & \\
\hline $15 / F$ & Father & $15 Y 10 \mathrm{M}$ & $15 Y 10 \mathrm{M}$ & $17 \mathrm{Y} 4 \mathrm{M}$ & $(1.0 x-3.50 /-0.5 \times 10)$ & $(1.0 x-3.0 /-0.5 \times 170)$ & & \\
\hline $16 / \mathrm{M}$ & Father & 14Y2M & 14Y2M & $16 \mathrm{Y} 2 \mathrm{M}$ & No data & No data & $\mathrm{ET}(\mathrm{Kr} 30)$ & \\
\hline $17 / \mathrm{M}$ & Mother & $9 Y 3 \mathrm{M}$ & $10 \mathrm{YMM}$ & $16 \mathrm{YM}$ & $(0.5 x+5.0 /-3.5 \times 0)$ & $(0.4 x+5.0 /-4.5 \times 165)$ & & $\mathrm{P}$ \\
\hline $18 / \mathrm{F}$ & Mother & $1 \mathrm{Y} 2 \mathrm{M}$ & $1 Y 4 \mathrm{M}$ & $1 Y 10 \mathrm{M}$ & No data & No data & ET, Ny & \\
\hline $19 / F$ & $\mathrm{~N}$ & $3 Y 5 M$ & $3 Y 6 M$ & $10 Y 10 \mathrm{M}$ & $(1.0 x+0.5)$ & $(1.0 x+0.5)$ & & \\
\hline $20 / \mathrm{F}$ & Father & $8 \mathrm{M}$ & $1 Y 4 \mathrm{M}$ & $7 \mathrm{Y} 4 \mathrm{M}$ & $(1.0 x+1.50)$ & $(1.0 x+1.25)$ & & \\
\hline $21 / \mathrm{M}$ & $\mathrm{N}$ & $2 Y$ & $2 Y 8 \mathrm{M}$ & $4 \mathrm{Y} 10 \mathrm{M}$ & $(0.3 x+2.5 /-2.5 \times 160)$ & $(0.3 x+2.0 /-2.0 \times 0)$ & & $\mathrm{P}$ \\
\hline $22 / \mathrm{F}$ & Father & $2 \mathrm{M}$ & $2 Y 1 \mathrm{M}$ & $19 \mathrm{YM}$ & $(0.8 x-6.5 /-0.75 \times 90)$ & $(1.0 x-1.0 /-1.0 \times 120)$ & & \\
\hline $23 / \mathrm{M}$ & $\mathrm{N}$ & $2 Y 8 \mathrm{M}$ & $3 Y$ & $22 Y$ & $(1.0 x-0.25)$ & $(1.0 x-0.5 /-2 \times 180)$ & & \\
\hline
\end{tabular}

$\mathrm{ET}=$ esotrophia; $\mathrm{FS}=$ frontalis suspension; $\mathrm{Kr}=$ Krimsky; $\mathrm{LR}=$ levator resection; $\mathrm{Ny}=$ nystagmus, $\mathrm{OP}=$ operation, $\mathrm{P}=$ positive; $\mathrm{XT}=\mathrm{exotrophia}$.

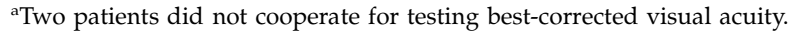

eye, $r=-0.3904, P=0.0655$; left eye, $r=-0.3534$,

$P=0.0981$ ). Detailed data are shown in Table 5 .

Complications of the one-stage procedures were mild. Although suture scars are a particularly important issue for patients in Asia because of keloid formation, scarring became less visible with age. All patients had temporary postoperative lagophthalmos, which gradually subsided. However, two patients (22\%) in the levator resection group had persistent lagophthalmos. There was no keratitis or corneal scarring. In two of 23 patients (8.7\%), the transnasal wiring loosened. There were no signs of infection with the wiring or lacrimal passage injury after transnasal wiring. Postoperative ectropion of the upper eyelid did not occur after levator resection. In addition, no stitch abscess or sign of infection occurred in patients who underwent frontalis suspension or levator resection.

Only two patients had major complications. Rarely, lateral canthotomy reduces the lateral support of the lower eyelid with disastrous consequences causing disfiguration of the lateral canthus and ectropion. This occurred in one patient, who underwent surgical correction of the condition. Malignant hyperthermia is a rare complication but associated with musculoskeletal ocular surgery such as ptosis surgery. Malignant hyperthermia occurred in one patient at the end of anaesthesia, and he died 3 days after surgery.

\section{Discussion}

\section{Outcome of one-stage correction for blepharophimosis syndrome}

One-stage surgical repair of blepharophimosis syndrome is currently advocated and yields satisfactory results. ${ }^{14,15}$ Nakajima et al ${ }^{14}$ described good surgical results using a procedure in which medial canthoplasty and levator resection were performed in one stage to treat 11 cases of blepharophimosis syndrome during 9 years. ${ }^{14}$ In 1994, Karacaoglan et $a l^{15}$ also performed medial canthoplasty, frontal suspension and nasal bone grafting in one stage to treat five patients with the syndrome during 2 years. However, these authors did not mention the severity of blepharophimosis syndrome and lacked objective evaluations. To the best of our knowledge, our study is the first to describe the degrees of the blepharophimosis syndrome, one-stage surgical management according to the severity and the outcomes. 
Table 2 Preoperative and postoperative data for 23 patients with blepharophimosis syndrome

\begin{tabular}{|c|c|c|c|c|c|c|c|c|}
\hline No/sex & $\begin{array}{l}\text { Preop } \\
\text { IICD }\end{array}$ & $\begin{array}{c}\text { Preop } \\
\operatorname{HPFL}(R, L)\end{array}$ & Ratio & $\begin{array}{l}\text { BPES } \\
\text { severity }\end{array}$ & OP method & $\begin{array}{l}\text { Postop } \\
\text { IICD }\end{array}$ & $\begin{array}{c}\text { Postop HPFL } \\
(R, L)\end{array}$ & Ratio \\
\hline \multicolumn{9}{|l|}{$L R$ group } \\
\hline $1 / \mathrm{F}$ & 35 & $(13,14)$ & 2.6 & Severe & $\mathrm{LC}+\mathrm{TW}+\mathrm{MP}$ & 28 & $(24,24)$ & 1.16 \\
\hline $2 / \mathrm{F}$ & 30 & $(15,15)$ & 2 & Severe & $\mathrm{LC}+\mathrm{TW}+\mathrm{MP}$ & 30 & $(25,25)$ & 1.2 \\
\hline $3 / F$ & 36 & $(15,15)$ & 2.4 & Severe & $\mathrm{LC}+\mathrm{TW}+\mathrm{MP}$ & 28 & $(22,22)$ & 1.27 \\
\hline $4 / \mathrm{M}$ & 40 & $(24,24)$ & 1.66 & Moderate & $\mathrm{TW}+\mathrm{MP}$ & 30 & $(30,30)$ & 1 \\
\hline $5 / \mathrm{M}$ & 37 & $(22,22)$ & 1.68 & Moderate & $\mathrm{TW}+\mathrm{MP}$ & 34 & $(30,30)$ & 1.16 \\
\hline \multicolumn{9}{|l|}{ FS group } \\
\hline $6 / \mathrm{M}$ & 38 & $(15,15)$ & 2.5 & Severe & $\mathrm{LC}+\mathrm{TW}+\mathrm{MP}$ & 33 & $(21,22)$ & 1.5 \\
\hline $7 / \mathrm{F}$ & 44 & $(21,21)$ & 2.1 & Severe & $\mathrm{LC}+\mathrm{TW}+\mathrm{MP}$ & 30 & $(23,23)$ & 1.3 \\
\hline $8 / \mathrm{F}$ & 34 & $(23,23)$ & 1.47 & Mild & MP & 34 & $(23,23)$ & $1.47^{\mathrm{a}}$ \\
\hline $9 / \mathrm{M}$ & 40 & $(15,15)$ & 2.67 & Severe & $\mathrm{LC}+\mathrm{TW}+\mathrm{MP}$ & 40 & $(20,20)$ & $2^{\mathrm{b}}$ \\
\hline $10 / \mathrm{F}$ & 35 & $(18,18)$ & 1.94 & Severe & $\mathrm{LC}+\mathrm{TW}+\mathrm{MP}$ & 27 & $(26,26)$ & 1.04 \\
\hline $11 / \mathrm{F}$ & 42 & $(19,20)$ & 2.1 & Severe & $\mathrm{LC}+\mathrm{TW}+\mathrm{MP}$ & 28 & $(30,30)$ & 0.93 \\
\hline $12 / \mathrm{F}$ & 35 & $(18,18)$ & 1.94 & Severe & $\mathrm{LC}+\mathrm{TW}+\mathrm{MP}$ & 32 & $(28,28)$ & 1.14 \\
\hline $13 / F$ & 37 & $(18,18)$ & 2.05 & Severe & $\mathrm{LC}+\mathrm{TW}+\mathrm{MP}$ & 26 & $(22,24)$ & 1.13 \\
\hline $14 / \mathrm{M}$ & 36 & $(20,20)$ & 1.8 & Moderate & $\mathrm{TW}+\mathrm{MP}$ & 32 & $(27,27)$ & 1.19 \\
\hline $15 / F$ & 47 & $(23,23)$ & 2.04 & Severe & $\mathrm{LC}+\mathrm{TW}+\mathrm{MP}$ & 40 & $(25,26)$ & $1.53^{\mathrm{b}}$ \\
\hline $16 / \mathrm{M}$ & 48 & $(23,23)$ & 2 & Severe & $\mathrm{LC}+\mathrm{TW}+\mathrm{MP}$ & 34 & $(26,26)$ & 1.3 \\
\hline $17 / \mathrm{M}$ & 42 & $(25,25)$ & 1.68 & Moderate & $\mathrm{TW}+\mathrm{MP}$ & 30 & $(31,31)$ & 1 \\
\hline $18 / \mathrm{F}$ & 36 & $(18,19)$ & 2 & Severe & $\mathrm{LC}+\mathrm{TW}+\mathrm{MP}$ & 30 & $(25,22)$ & 1.2 \\
\hline $19 / \mathrm{F}$ & 36 & $(19,19)$ & 1.89 & Severe & $\mathrm{LC}+\mathrm{TW}+\mathrm{MP}$ & 32 & $(23,23)$ & 1.39 \\
\hline $20 / F$ & 40 & $(15,15)$ & 2 & Severe & $\mathrm{LC}+\mathrm{TW}+\mathrm{MP}$ & 32 & $(27,27)$ & 1.19 \\
\hline $21 / \mathrm{M}$ & 35 & $(20,20)$ & 1.75 & Moderate & $\mathrm{TW}+\mathrm{MP}$ & 30 & $(27,27)$ & 1.11 \\
\hline $22 / \mathrm{F}$ & 42 & $(19,19)$ & 2.2 & Severe & $\mathrm{LC}+\mathrm{TW}+\mathrm{MP}$ & 40 & $(35,35)$ & 1.14 \\
\hline $23 / \mathrm{M}$ & 33 & $(16,17)$ & 2 & Severe & $\mathrm{LC}+\mathrm{TW}+\mathrm{MP}$ & 25 & $(26,26)$ & 1 \\
\hline
\end{tabular}

$\mathrm{FS}=$ frontalis suspension; $\mathrm{HPFL}=$ horizontal palpebral fissure length; IICD = inner intercanthal distance; LC $=$ lateral canthoplasty; $\mathrm{LR}=$ levator resection; $\mathrm{MP}=$ medial canthoplasty; $\mathrm{TW}=$ transnasal wiring.

apostoperative blepharophimosis ratio is the same as preoperative.

blepharophimosis ratio greater than 1.5 as poor result.

Table 3 Statistical data for 23 patients with blepharophimosis syndrome

\begin{tabular}{lccr}
\hline$I C D(\mathrm{~mm})$ & Pre-operative & Postoperative & P-value \\
\hline Mean \pm SD (range) & $38.17 \pm 4.45(30-48)$ & $31.52 \pm 4.17(25-40)$ & $P<0.0001$ \\
HPFL (mm) & $18.96 \pm 3.3(13-25)$ & $25.93 \pm 3.56(20-35)$ & \\
Mean \pm SD (range) & & & \\
Blepharophimosis (ratio $=$ IICD/HPFL) & 2 & 1.23 \\
Mean (ratio) & $0(0)$ & $16(70)$ \\
Normal $(<1.3)(n(\%))$ & $1(4)$ & $5(22)$ \\
Mild $(1.3-1.5)(n(\%))$ & $5(22)$ & $1(4)$ & \\
Moderate $(1.5-1.8)(n(\%))$ & $17(74)$ & & \\
Severe $(>1.8)(n(\%))$ & &
\end{tabular}

$\mathrm{HPFL}=$ horizontal palpebral fissure length; IICD $=$ inner intercanthal distance.

We chose the ratio between the IICD and HPFL as the measure of the degree of blepharophimosis severity, as the IICD and HPFL will elongate as the patients grow. It is very difficult to evaluate the long-term effect of surgical correction using a single measurement of the IICD or HPFL. In our study, 16 of $23(70 \%)$ patients had good outcomes, with ratios less than 1.3 postoperatively. Only two of $23(8.7 \%)$ patients had the transnasal wiring loosened, which could be secondary to cheese wiring of the wire through the soft tissues. One patient with mild blepharophimosis underwent only medial canthoplasty and frontalis suspension. The postoperative

blepharophimosis ratio of this patient was the same as preoperative ratio. Only medial canthoplasty seems not to improve the blepharophimosis ratio, but is helpful for improving patient appearance.

When ptosis surgery and medial canthopexy are performed at the same time, the vertical and horizontal 
lengths pull against each other. This results in the development of excessively strong traction in different directions on the palpebral fissure. There are high risks in
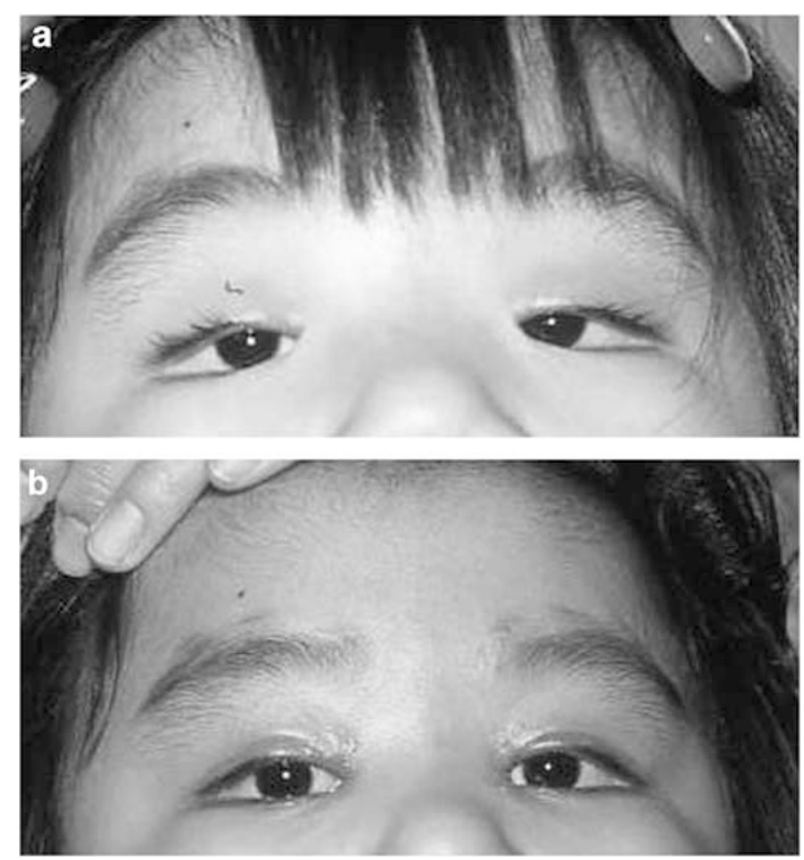

Figure 2 (a) Preoperative view of a 4-year-old girl (patient 8) with blepharophimosis syndrome; mild blepharophimosis $($ ratio $=1.47)$. (b) Same patient's appearance 6 months after one-stage surgical correction. developing insufficiency or loosening of the medial canthopexy and poor elevation of IPFH. For this reason, we designed the intercanthal distance and added $4 \mathrm{~mm}$
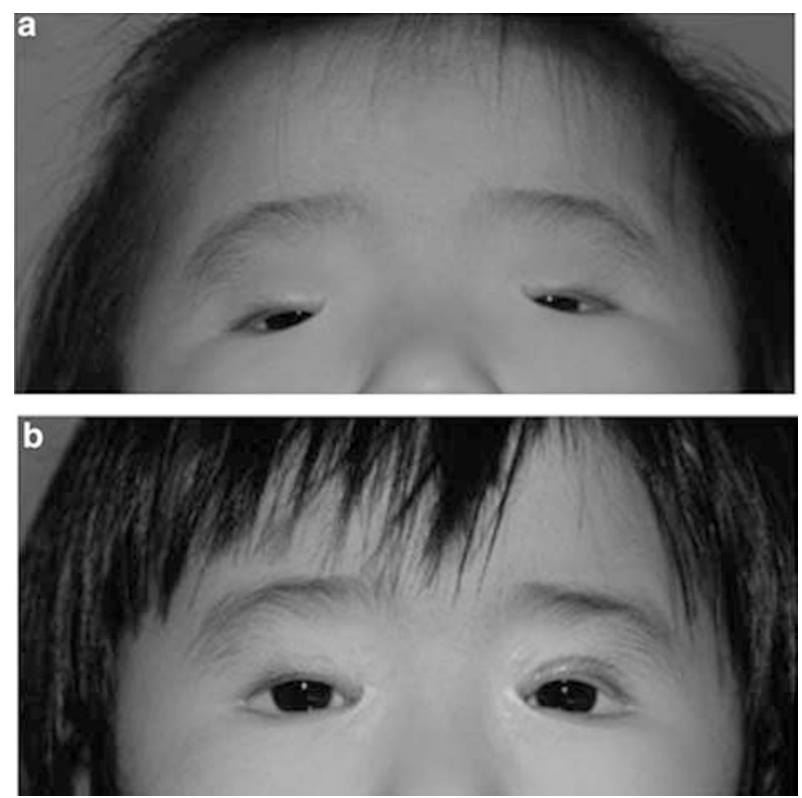

Figure 4 (a) Preoperative view of a 2-year-old girl (patient 3) with blepharophimosis syndrome; the IPFH measures $2 \mathrm{~mm}$. (b) Same patient's appearance 1-year postoperatively.
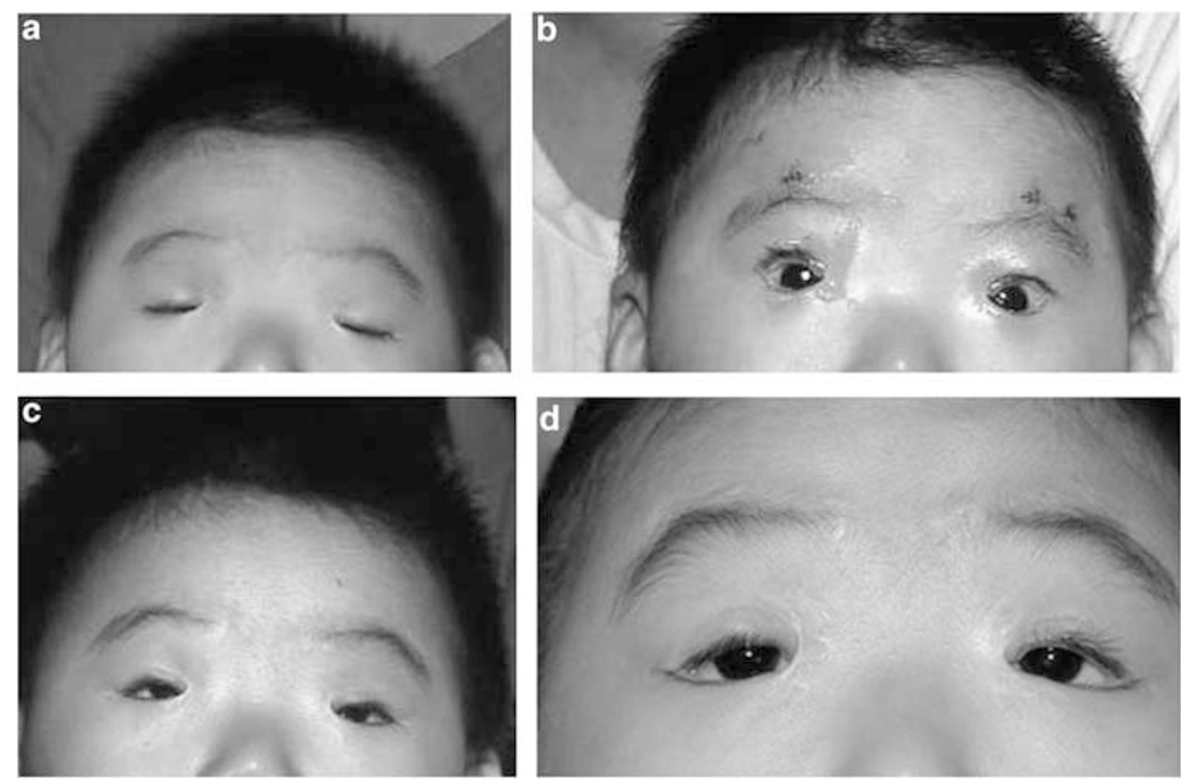

Figure 3 (a) Preoperative view of a 5-month-old boy (patient 6) with blepharophimosis syndrome; the IPFH measures $1 \mathrm{~mm}$. (b) The same patient's appearance 1 day after frontalis suspension with Supramid (S Jackson Inc., Alexandria, VA, USA). (c) Same patient's appearance 8 months after frontalis suspension; 4 months before one-stage correction. (d) Poor elevation of the left interpalpebral fissure occurred after one-stage correction. Then, he underwent levator resection of the left eye 1-year after the one-stage procedures. Same patient's appearance 6 months after levator resection of left eye. 
Table 4 Preoperative and postoperative ptosis data for 23 patients with blepharophimosis syndrome

\begin{tabular}{|c|c|c|c|c|c|c|c|c|}
\hline No & $\begin{array}{c}\text { Age at } \\
O P\end{array}$ & $\begin{array}{l}\text { Preop } \\
\text { IPHF } \\
(R, L)\end{array}$ & $\begin{array}{c}L M F \\
(R, L)\end{array}$ & $\begin{array}{c}\text { Follow-up } \\
\text { duration } \\
\text { (months) }\end{array}$ & $\begin{array}{l}\text { Postop } \\
\text { IPHF } \\
(R, L)\end{array}$ & $\begin{array}{l}\text { Lid level } \\
\text { results at last } \\
\text { visit }\end{array}$ & Reoperation & $\begin{array}{l}\text { Prior } \\
O P\end{array}$ \\
\hline \multicolumn{9}{|c|}{ LR group } \\
\hline 1 & $3 Y$ & $(3,3)$ & $(\mathrm{P}, \mathrm{P})$ & 6 & $(7,7)$ & Moderate & & \\
\hline 2 & $2 Y 7 M$ & $(2,2)$ & $(\mathrm{P}, \mathrm{P})$ & 18 & $(6,6)$ & Moderate & & \\
\hline 3 & $2 Y 5 \mathrm{M}$ & $(2,2)$ & $(\mathrm{P}, \mathrm{P})$ & 24 & $(7,7)$ & Moderate & & \\
\hline 4 & $2 Y 5 \mathrm{M}$ & $(3,3)$ & $(\mathrm{P}, \mathrm{P})$ & 24 & $(6,6)$ & Moderate & & \\
\hline 5 & $3 Y 7 M$ & $(3,3)$ & $(\mathrm{P}, \mathrm{P})$ & 20 & $(6,6)$ & Moderate & & \\
\hline \multicolumn{9}{|c|}{ FS group } \\
\hline 6 & $1 Y 8 M$ & $(1,1)$ & $(\mathrm{P}, \mathrm{P})$ & 37 & $(6,3)$ & Poor & LR (OS) & FS, Supramid \\
\hline 7 & $8 Y 6 \mathrm{M}$ & $(4,3)$ & $(\mathrm{P}, \mathrm{P})$ & 12 & $(8,5)$ & Poor & & \\
\hline 8 & $4 Y 8 \mathrm{M}$ & $(6,5)$ & $(\mathrm{P}, \mathrm{P})$ & 6 & $(8,8)$ & Good & & \\
\hline 9 & $1 Y 4 M$ & $(1,1)$ & $(\mathrm{P}, \mathrm{P})$ & 104 & $(3,3)$ & Poor & FS (OU) & \\
\hline 10 & $3 Y 2 M$ & $(4,4)$ & $(\mathrm{P}, \mathrm{P})$ & 97 & $(6,6)$ & Moderate & & \\
\hline 11 & $20 Y 7 M$ & $(4,5)$ & $(\mathrm{P}, \mathrm{P})$ & 6 & $(6,6)$ & Moderate & & \\
\hline 12 & 4Y1M & $(3,3)$ & $(\mathrm{P}, \mathrm{P})$ & 10 & $(5,5)$ & Moderate & & \\
\hline 13 & $2 Y 3 M$ & $(3,4)$ & $(\mathrm{P}, \mathrm{P})$ & 16 & $(7,7)$ & Moderate & & \\
\hline 14 & $2 \mathrm{Y} 4 \mathrm{M}$ & $(2,2)$ & $(\mathrm{P}, \mathrm{P})$ & 36 & $(6,6)$ & Moderate & & \\
\hline 15 & $15 Y 10 \mathrm{M}$ & $(4,4)$ & $(\mathrm{P}, \mathrm{P})$ & 18 & $(6,6)$ & Moderate & & \\
\hline 16 & $14 \mathrm{Y} 2 \mathrm{M}$ & $(1,2)$ & $(\mathrm{P}, \mathrm{P})$ & 24 & $(3,4)$ & Poor & & \\
\hline 17 & $10 Y 3 M$ & $(4,3)$ & $(\mathrm{P}, \mathrm{P})$ & 72 & $(7,6)$ & Moderate & & \\
\hline 18 & $1 Y 4 M$ & $(1,2)$ & $(\mathrm{P}, \mathrm{P})$ & 6 & $(4,6)$ & Poor & & \\
\hline 19 & $3 Y 6 M$ & $(4,3)$ & $(\mathrm{P}, \mathrm{P})$ & 92 & $(5.5,5.5)$ & Moderate & & \\
\hline 20 & $1 Y 4 M$ & $(2,2)$ & $(\mathrm{P}, \mathrm{P})$ & 72 & $(7,6)$ & Moderate & & \\
\hline 21 & $2 Y 8 M$ & $(3,3)$ & $(\mathrm{P}, \mathrm{P})$ & 26 & $(7,7)$ & Moderate & & \\
\hline 22 & $2 \mathrm{Y} 1 \mathrm{M}$ & $(2,2)$ & $(\mathrm{P}, \mathrm{P})$ & 204 & $(5,4)$ & Poor & FS (OU) & \\
\hline 23 & $3 Y$ & $(1,1)$ & $(\mathrm{P}, \mathrm{P})$ & 228 & $(2.5,2.5)$ & Poor & LR (OU) & \\
\hline
\end{tabular}

$\mathrm{FS}=$ frontalis suspension; $\mathrm{IPFH}=$ interpalpebral fissure height; $\mathrm{LR}=$ levator resection; $\mathrm{LMF}=$ levator muscle function; $\mathrm{OU}=$ both eye; $\mathrm{OS}=$ left eye; $\mathrm{OD}=$ right eye; $\mathrm{P}=$ poor.

Table 5 Relationship between severity of blepharophimosis and ptosis

\begin{tabular}{llll}
\hline Spearman correlation & \multicolumn{1}{c}{ Right PFH } & \multicolumn{1}{c}{ Left PFH } & No significance \\
\hline IICD & $\rho=0.0397, P=0.8547$ & $\rho=0.0308, P=0.8892$ & Significance \\
Right HPFL & $\rho=0.4997, P=0.0152^{*}$ & & Significance \\
Left HPFL & $\rho=-0.3904, P=0.0655$ & $\rho=0.4839, P=0.0193^{*}$ & Borderline \\
Ratio & $\rho=0.3534, P=0.0981$ & \\
\hline
\end{tabular}

$\mathrm{HPFL}=$ horizontal palpebral fissure length; IICD = inner intercanthal distance; $\mathrm{PFH}=$ palpebral fissure height. ${ }^{*} P<0.05$.

for overcorrection using a transnasal wiring procedure to reduce the possibility of reappearance of telecanthus. Otherwise, the fissure opened in ptosis surgery tends to narrow back after medial canthoplasty. It is very difficult to adjust and elevate the IPFH during the operation. We did our best to create a good contour and symmetrical eyelid by maximal levator resection or frontal suspension. About 1 week after surgery, the palpebral fissure widened and gradually became apparent to yield a good shape. The cosmetic results were acceptable to most of the patients.

Our patients had no or minimal levator function. Therefore, frontalis suspension was indicated in all of our patients. Autogenous fascia lata is the most appropriate material for use in frontalis suspension, but there are insufficient amounts of this material and scarring results in children younger than 3 years of age. ${ }^{26}$ Using donor fascia lata, a second surgical site for obtaining autogenous fascia, with the attendant morbidity, is avoided. ${ }^{27}$ Furthermore, donor fascia reduces the complexity of the surgical procedure for one-stage correction. Donor fascia lata provides a reasonable alternative material for frontalis suspension and does not have some of the disadvantages of synthetic materials, such as extrusion, granuloma formation, and infection. ${ }^{28-30}$ The early stage, all patients underwent 
frontalis suspension with donor fascia lata. Of the 18 patients who underwent frontalis suspension, only two (11\%) with palpebral fissure heights (PFHs) more than $2 \mathrm{~mm}$ underwent reoperation for residual ptosis and asymmetrical eyelid positions. In contrast, all five (100\%) patients with PFHs less than $2 \mathrm{~mm}$ (very severe ptosis) needed reoperations. As a result, we conclude that patients with very severe ptosis are not good candidates for one-stage blepharophimosis procedures. There is a strong possibility of poor elevation of the IPFH.

As levator resection is more physiologic, with a better eyelid crease and involves no brow scar, we commenced treatment with maximal levator resection since 2002. Although anterior levator resection is the preferred procedure used to correct ptosis in patients with at least $4 \mathrm{~mm}$ of levator function, Blomgren and Holmstrom ${ }^{31}$ suggested that anterior levator resection can be used for all types of congenital ptosis, and especially in severe ptosis. In addition, Mauriello et $a^{25}$ in 1985 described maximal levator resection is indicated for severe congenital ptosis and poor to absent levator function. In their study, $28(87.5 \%)$ patients had successful results, which were achieved if the lid position covered $3 \mathrm{~mm}$ or less of cornea superiorly. In our study, none of the five patients with maximal levator resection needed reoperation and all five patients had moderately good results. However, the IPFH in all of these patients was greater than $2 \mathrm{~mm}$ and the follow-up period was not long (mean, 18 months). The effect of levator resection needs further observation.

Malignant hyperthermia is an uncommon but lifethreatening complication. There may be an increased likelihood of malignant hyperthermia for patients with musculoskeletal ocular disorders. ${ }^{32}$ One patient in our study had malignant hyperthermia and died 3 days after surgery despite intensive medical care (early on in the study period). The one-stage procedure is a relatively long surgery and increases the risk of malignant hyperthermia. Henceforth, when we performed onestage surgery for correction of blepharophimosis syndrome, we carefully monitored muscle tone and body temperature to avoid malignant hyperthermia. Surgery should be terminated immediately if malignant hyperthermia is suspected. Currently, due to clinical and research investigation and widespread education, mortality from malignant hyperthermia is less than $5 \% .^{33}$

\section{Timing and indications for one-stage blepharophimosis surgery}

Traditional management of blepharophimosis syndrome includes medial canthoplasty between the ages of 3 and 5 years, followed by ptosis correction about 6 months later. ${ }^{12,13}$ However, patients with blepharophimosis syndrome have a high rate of amblyopia. ${ }^{7}$ In 2003, Beckingsale et $a l^{34}$ recommended that patients with severe ptosis have it corrected before 3 years of age, and that all other patients should undergo surgery before 5 years of age. Traditional multiple surgeries may prolong the treatment course and most importantly, it may delay the amblyopia management and influence the visual outcome. In our study, 13 of $18(72 \%)$ patients underwent one-stage surgery before the age of 3 years and 18 of 23 $(78 \%)$ patients underwent one-stage surgery before the age of 5 years. Of the 18 patients who underwent the operation before 5 years of age, five lacked data for bestcorrected vision. Four patients (31\%) had amblyopia. The percentage of amblyopia was lower as compared with previous studies, possibly due to very early treatment. ${ }^{7,34}$

Now, many surgeons suggest correction of ptosis first, even at a very early age, to prevent amblyopia. Softtissue medial canthal and lateral canthal surgery can wait until the face is grown. Although in the very young (less than 3 years) the main indication for surgery was threat to visual development in this blepharophimosis cohort, cosmesis was the predominant indication for surgery. In our experience, many parents preferred correcting all abnormalities of blepharophimosis syndrome before the child commenced primary school at around the age of 5 years. Furthermore, according to the statistical results, the lower palpebral apertures tended to have larger blepharophimosis ratios, requiring correction. Therefore, regarding the patients with the palpebral apertures greater than $2 \mathrm{~mm}$, one-stage correction provides acceptable results both in functional and cosmetic improvements and obviates the need for two surgeries. The risks and expenses associated with two separate surgeries are decreased, hospitalisation time is potentially reduced, and subsequent rehabilitation can be initiated in a more timely fashion. In contrast, patients with very severe ptosis are at greater risk for development of amblyopia and poor results for one-stage blepharophimosis correction. Thus, we suggest correction of ptosis first, even at a very early age for the prevention of amblyopia, followed by correction of telecanthus and small HPFL at an older age.

\section{Conclusions}

Blepharophimosis syndrome (BPES) is a complex of multiple eyelid malformations. Considering our results with previous reports in the literature, we believe that patients with mild to moderate blepharophimosis syndrome (IPFH more than $2 \mathrm{~mm}$ ) will benefit from the one-stage operation. ${ }^{14,15,30,35}$ Depending on the statistical analysis, patients with mild to moderate ptosis usually had milder blepharophimosis. For patients with clear visual axes, corrective surgery could wait until 3-5 years 
of age. Depending on levator function, the surgeon could choose medial canthoplasty with frontalis suspension using autogenous fascia lata or anterior levator resection. For patients with obscured visual axes but with IPFHs more than $2 \mathrm{~mm}$, the one-stage correction can be performed when the child is less than 3 years of age. However, in cases with very severe ptosis (the IPFH less than $2 \mathrm{~mm}$ ), multiple stages of reconstruction are advised and should be treated as early as possible. Nonetheless, future prospective controlled studies may still be necessary to evaluate the benefits of one-stage repair $v s$ staged surgery in blepharophimosis patients. If ours and others' results are confirmed in prospective controlled studies, one-stage correction would decrease the number of total procedures and reduce the time needed for surgical rehabilitation.

\section{References}

1 Owens N, Hadley R, Kloepfer HW. Hereditary blepharophimosis, ptosis and epicanthus inversus. J Int Coll Surg 1960; 33: 558-574.

2 Kohn R, Romano PE. Blepharoptosis, blepharophimosis, epicanthus inversus, and telecanthus-A syndrome with no name. Am J Ophthmol 1971; 72: 625-632.

3 Zlotogora J, Sagi M, Cohen T. The blepharophimosis, ptosis, and epicanthus inversus syndrome: delineation of two types. Am J Hum Genet 1983; 35: 1020-1027.

4 Oley C, Baraitser M. Blepharophimosos, ptosis, epicanthus inversus syndrome (BPES syndrome). J Med Genet 1988; 25: $47-51$.

5 Stromme P, Sandboe F. Blepharophimosis-ptosis-epicanthus inversus syndrome (BPES). Acta Ophthalmol Scand 1996; 74: $45-47$.

6 Garden JW. Blepharophimosis, ptosis, epicanthus inversus and lacrimal stenosis. Am J Ophthmol 1969; 67: 153-154.

7 Beaconsfield M, Walker JW, Collin JRO. Visual development in the blepharophimosis syndrome. Br J Opthalmol 1991; 75: 746-748.

8 Udar N, Yellore V, Chalukya M, Yelchits S, Silva-Garcia R, Small K. Comparative analysis of the FOXL2 gene and characterization of mutations in BPES patients. Hum Mut 2003; 22: 222-228.

9 Crisponi L, Deiana M, Loi A, Chiappe F, Uda M, Amati P et al. The putative forkhead transcription factor FOXL2 is mutated in blepharophimosis/ptosis/epicanthus inversus syndrome. Nat Genet 2001; 27: 159-166.

10 Hargrave M, James K, Nield K, Toomes C, Georgas K, Sullivan $\mathrm{T}$ et al. Fine mapping of the neurally expressed gene SOX14 to human 3q23, relative to three congenital diseases. Hum Genet 2000; 106: 432-439.

11 Amati P, Chomel JC, Nivelon-chevalier A, Gilgenkrantz S, Kitzis A, Kaplan J et al. A gene for blepharophimosis-ptosisepicanthus inversus syndrome maps to chromosome 3q23. Hum Genet 1995; 96: 213-215.

12 Johnson CC. Surgical repair of the syndrome of epicanthus inversus, blepharophimosis and ptosis. Arch Ophthalmol 1964; 71: 510-516.
13 Elliot D, Wallace AF. Ptosis with blepharophimosis and epicanthus inversus. Br J Plast Surg 1986; 39: 244-248.

14 Nakajima T, Ypshimura Y, Onishi K, Sakakibara A. Onestage repair of blepharophimosis. Plast Reconstr Surg 1991; 87: $24-31$

15 Karacaoglan N, Sahin U, Ercan U, Bozdogan N. One-stage repair of blepharophimsis: a new method. Plast Reconstr Surg 1994; 93: 1406-1409.

16 Mustarde' JC. Epicanthus and telecanthus. Br J Plast Surg 1963; 16: 345-356.

17 Mustarde' JC. Epicanthus folds and problem of telecanthus. Trans Ophthalmol Soc UK 1963; 83: 397-411.

18 Anderson RL, Baumgartner SA. Amblyopia in ptosis. Arch Ophthalmol 1980; 98: 1068-1069.

19 Freuh HR. The mechanistic classification of ptosis. Arch Ophthalmol 1980; 87: 1019-1021.

20 Gupta VP, Sodhi PK, Pandey RM. Normal values for inner intercanthal, interpupillary, and outer intercanthal distance in the Indian population. Int J Clin Pract 2003; 57 25-29.

21 Quant JR, Woo GC. Normal values of eye position in the Chinese population in Hong Kong. Optom Vision Sci 1992; 69: 152-158.

22 Quant JR, Woo GC. Normal values of eye position and head size in Chinese children from Hong Kong. Optom vision Sci 1993; 70: 668-671.

23 Manners RM, Tyers AG, Morris RJ. The use of prolene as a temporary suspensory material for brow suspension in young children. Eye 1994; 8: 346-348.

24 Fox SA. Congenital ptosis. II. Frontalis sling. J Pediatric Ophthalmol 1966; 3: 25-28.

25 Mauriello JA, Wagner RS, Caputo AR, Natale B, Lister M. Treatment of congenital ptosis by maximal levator resection. Ophthalmology 1985; 93: 466-469.

26 Deenstra W, Melis P, Kon M, Werker P. Correction of severe blepharoptosis. Ann Plast Surg 1996; 36: 348-353.

27 Wheatcroft SM, Vardy SJ, Tyers AG. Complications of fascia lata harvesting for ptosis surgery. Br J Ophthalmol 1997; 81: 581-583.

28 Wasserman BN, Sprunger DT, Helveston EM. Comparison of material used in frontalis suspension. Arch Ophthalmol 2001; 119: 687-691.

29 Wilson ME, Johnson RW. Congenital ptosis Long-term results of treatment using lyophilized fascia lata for frontalis suspensions. Ophthalmology 1991; 98: 1234-1237.

30 Esmaeli B, Chung H, Pashby RC. Long-term results of frontalis suspension using irradiated, banked fascia lata. Ophthalmic Plast Reconstr Surg 1998; 14: 159-163.

31 Blomgren I, Holmstrom H. Anterior levator resection in congenital genuine blepharoptosis. A follow-up of 55 operated eyelids. Scand J Plast Reconstr Surg 1986; 20: 189-195.

32 Beasley H. Hyperthermia associated with ophthalmic surgery. Am J Ophthalmol 1974; 77: 76-79.

33 Ali SZ, Taguchi A, Rosenberg H. Malignant hyperthermia. Best Pract Res Clin Anesthesiol 2003; 17: 519-533.

34 Beckingsale PS, Sullivan TJ, Wong VA. Blepharophimosis: a recommendation for early surgery in patients with severe ptosis. Clin Exper Ophthalmol 2003; 31: 138-142.

35 Karacaoglu E, Tezel E, Guler MM. Rotation ligmentoplasty for the correction of epicanthus inversus. Ann Plast Surg 2000; 45: 140-144. 